\title{
Heart Rate Changes Before, During, and After Treadmill Walking Exercise in Normal Dogs
}

\author{
Sarah A. Shull ${ }^{1 *}$, Sarah K. Rich ${ }^{1}$, Robert L. Gillette ${ }^{2}$ and Jane M. Manfredi ${ }^{1}$ \\ ${ }^{1}$ College of Veterinary Medicine, Michigan State University, East Lansing, MI, United States, ${ }^{2}$ Sportsvet Veterinary Consulting \\ Services, Lancaster, SC, United States
}

\section{OPEN ACCESS}

Edited by:

David Levine,

University of Tennessee at

Chattanooga, United States

Reviewed by:

Michael Conzemius,

University of Minnesota Twin Cities,

United States

Denis J. Marcellin-Little,

University of California, Davis,

United States

Darryl L. Millis,

University of Tennessee, Knoxville,

United States

*Correspondence:

Sarah A. Shull

shulldvm@msu.edu

Specialty section:

This article was submitted to

Veterinary Surgery and

Anesthesiology,

a section of the journa

Frontiers in Veterinary Science

Received: 15 December 2020

Accepted: 12 February 2021

Published: 12 April 2021

Citation:

Shull SA, Rich SK, Gillette RL and Manfredi JM (2021) Heart Rate

Changes Before, During, and After

Treadmill Walking Exercise in Normal

Dogs. Front. Vet. Sci. 8:641871.

doi: 10.3389/fvets.2021.641871
In dogs, changes in heart rate (HR) can reflect conditioning, fear, anticipation, and pain; however, these are not routinely assessed in veterinary rehabilitation patients. Knowing the expected HR changes during rehabilitation exercises can guide protocols and can optimize post-operative therapy. The primary objectives of the study were to assess $\mathrm{HR}$ in dogs undergoing treadmill exercise (TE) during the walk and to compare the three collection techniques of HR, namely, auscultation, a HR monitor (HR MONITOR), and a Holter monitor (HOLTER). We hypothesized that the HR would increase by $20 \%$ during TE, that HR taken after TE would not be the same as the HR during TE, and that all methods of measurement would have good agreement. HR was recorded in all methods simultaneously, in eight adult healthy large breed dogs during rest (REST), immediately before TE (PRE), during TE (WALK), and 15 and 60 s after TE (POST-15, POST-60). Statistical analyses included Spearman and Pearson correlations, BlandAltman analyses, and a repeated measures ANOVA with Sidak's post-hoc test (significant at value of $p<0.05$ ). Increased HR was reflected in TE during WALK, and elevations in HR during WALK were not reflected in POST timepoints. Auscultation was also not possible during WALK. Significant moderate-to-strong correlations existed among all monitoring options at each of the timepoints (rho range $=0.5-0.9, p<0.05$ ). There were no correlations between peak HR and age or weight. The main limitation of this study is that only healthy and large breed dogs were used. Both monitors captured the increase in HR during exercise and could guide TE regimens to minimize patient risk of injury and to maximize training effectiveness.

Keywords: cardiovascular, monitoring, holter, dog, fitness, auscultation, rehabilitation

\section{INTRODUCTION}

Heart rate (HR) has historically been used to assess physical state and the effects of the type and intensity of exercise in the fields of human sports medicine and exercise science (1-3). Auscultation and electrocardiogram (ECG) readings are two traditionally used methods to compile HR data. More recently, HR monitors have become a standard method to acquire HR data, which is used as a training aid for a variety of sports (4-9). In the rehabilitation field, measuring the patient's HR is recommended by the American Physical Therapy Association (APTA) Guide to Physical Therapist Practice (10), although gathering this information may be infrequent and underused $(11,12)$. HR data can be used during exercise sessions to assess the efforts, cardiovascular status, pain, and recovery speed of the patient. 
Therapeutic exercise is commonly used in veterinary rehabilitation $(13,14)$. While HR during exercise has been examined in athletic dogs, there are gaps in understanding what to expect, and data are lacking for rehabilitation patients (15-23). Swanson et al. (19) developed a visual exertion scale for healthy dogs walking and trotting on a treadmill, finding that an increase in exertion correlated to an increase in HR. The authors recognized that larger studies were needed to validate their subjective measures and that the Holter monitor used in that study may be prohibitively expensive to those in private practice (19). Boddy et al. (20) described collecting HR by auscultation in a 6-min walk test in dogs with congestive heart failure as compared to normal healthy dogs: though post-walk HR did not significantly increase from baseline, distance traveled in the heart failure group was less. Swimmer and Rozanski (21) described using a 6-min walk test, including HR measurement performed in healthy dogs and dogs with pulmonary disease. The HR was measured before the walk but not during the exercise. The dogs were walked up and down a hallway, and not on a treadmill. The technique used to acquire the HR was not described, but it is assumed to be by auscultation as they referred to the study of Boddy et al. (20), and an increase in HR from baseline was seen post-walk in the pulmonary disease group, along with a shorter walk distance.

Although HR has shown to be a sound measurement of physiological status, the information on how the veterinary rehabilitation professional can best obtain and use it is lacking. For clinically useful HR monitoring in a practice setting, the approach must produce valid information, be economical, and be easy to apply and interpret in real time. An additional requirement for rehabilitation practices is the ability of monitoring technique to work while on an underwater treadmill and during exercise. Auscultation using a stethoscope is economical and easy to apply and interpret while the patient is stationary but has not been examined during rehabilitative work. Various Holter monitors have been used in clinical settings on cardiac patients, but their economical use, ease of setup, and ability to assess data in real time during exercise may limit their feasibility in practice (22-25). A HR monitor has been examined previously in dogs under stationary and trot conditions $(26,27)$; however, the validity and HR range in normal dogs during TE (WALK) using a different waterproof HR monitor model have not been assessed $(26,27)$. The walk is commonly used in rehabilitation settings, as patients are most often recovering from musculoskeletal or neurologic diseases making a faster pace unsafe. Establishing a normal HR variation and recovery time at the walk is key to tailoring rehabilitation regimens and assessing the progress of the patient. An ideal monitoring system for HR in dogs to be used in the veterinary rehabilitation setting has not been established.

It is also essential to recognize all the relative influences on HR. In dogs, HR has been examined as a physiologic parameter of conditioning, anticipation, fear, and pain, yet it is not routinely assessed in veterinary rehabilitation patients (28-32). Rehabilitative therapies have the potential to elicit stressful responses associated with the anticipation of an exercise or loud noise (treadmill turning on and water rushing in). In the previous studies, stress in the form of loud noises has shown an increased HR in dogs of 15-50 beats per minute (bpm), and stress in the form of anticipation has also elevated HR a mean of $43 \mathrm{bpm}(28,29)$. HR elevations in these instances suggest a level of stress that may not benefit healing (33-38). In humans, elevated HR has been demonstrated in chronic pain cases, where many of our veterinary rehabilitation patients also experience chronic pain $(36,37)$. Given that rehabilitation exercises are performed in dogs often with chronic pain or recovering from surgery, distinguishing normal HR elevations and recovery time due to TE from other causes is essential for guiding clinical care to minimize pain and maximize conditioning opportunities.

Knowing the expected normal changes in $\mathrm{HR}$ and HR recovery during rehabilitation exercises can guide protocols to optimize therapy and conditioning while monitoring the progress of the patient. Changes in patient pain and stress levels reflected by HR changes can dictate post-operative patient care orders by setting parameters that would indicate the need to decrease the intensity of exercise (by slowing the speed) or its duration. A faster HR recovery is a positive prognostic indicator of recovery in dogs and humans with cardiovascular disease (3945). Therefore, the primary objectives of the study were to assess the HR in healthy dogs undergoing treadmill exercise (TE) and to compare the feasibility of using each of the three HR data collection techniques, namely, auscultation, a HR monitor, and a Holter monitor, in a practice setting. We hypothesized that HR would increase by $20 \%$ from baseline during TE, that HR taken 15 and $60 \mathrm{~s}$ after TE would not reflect HR during TE, and that all measurement methods would have good agreement and prove feasible.

\section{MATERIALS AND METHODS \\ Dogs}

This study was compliant with the National Institutes of Health (NIH) guidelines for Humane Care and Use of Animals and was approved by our institutional animal care and use committee with proper consent forms filled out. Eight clientowned dogs were used, with three intact males (one German Wirehaired Pointer, one Curly-Coated Retriever, and one Labrador Retriever), one neutered male (mixed breed), one intact female (Labrador Retriever), and three spayed females (CurlyCoated Retrievers). The average age of the dogs was 52 (SD \pm 46) months (with all dogs being between 7 months and 10 years of age), weight was $29.1(\mathrm{SD} \pm 3.7) \mathrm{kg}$, and body condition score was $4-5 / 9$. All dogs were previously acclimated to the treadmill and treadmill room and had completed three 6-min walks on the treadmill within 3 months prior to the start of this study. Dogs were examined and found to be generally healthy, based on a general, orthopedic, and neurologic examination performed by the veterinarian who is in charge of the rehabilitation unit of the hospital. None of the dogs had a history or current evidence of cardiovascular, neurologic, or musculoskeletal disease. The medical records for the dogs were available, and none of the dogs had a previous diagnosis of osteoarthritis or other musculoskeletal diseases which would interfere with this study. The regular exercise routine of the dog included walking for 
$1.6 \mathrm{~km}$ for $\sim 15 \mathrm{~min}$, four times a week, for 3 months before the start of the study. No specific conditioning training was performed for this study.

\section{Heart Rate Monitors and Placement}

A Holter monitor (HOLTER; Dextronix, Sacramento, CA, USA) and a HR monitor (HR MONITOR; Polar USA, Bethpage, NY, USA) were used to assess HR in addition to auscultation with a stethoscope. Both leads and pads of the HOLTER and the HR MONITOR were waterproof, with their electronics completely encased in plastic so water would not interfere with readings $(46,47)$. For HOLTER placement, a $3 \times 3 \mathrm{~cm}$ area of hair was clipped, and the skin was cleaned with alcohol in three areas, one over the sternum and one each over the ventral left and right thoraces, before each of the three HOLTER ECG pads was applied. The hair caudal to the HOLTER on the ventrolateral aspect on the left chest of the dog was wet with alcohol before the placement of the single HR MONITOR pad. Both took $5 \mathrm{~min}$ to place in total and were secured with tape and a Polar strap over the handle of a Help'EmUp harness (Figure 1). This setup allowed for simultaneous recordings of HR with both systems while also allowing auscultation. The HOLTER monitor provided ECG tracings of HR over time. The HR MONITOR provided a constantly updating number indicating the current HR in beats per minute on its visual display and did not display or record an ECG-like tracing. The experiment occurred in a quiet room with a temperature between 18 and $22^{\circ} \mathrm{C}$. Dogs were fasted for at least $2 \mathrm{~h}$ before the experiment.

\section{Heart Rate Recordings}

The treadmill (Companion Underwater Treadmill, Ft. Wayne, IN, USA) used for this study had no water present and 0 degrees of incline (Figure 2). HR was recorded in 1-min time intervals before TE after being in the treadmill room for $5 \mathrm{~min}$ (REST), immediately before TE while inside the non-moving treadmill (PRE), while walking on the treadmill (WALK) for $4 \mathrm{~min}$, and $15 \mathrm{~s}$ (POST-15) and $60 \mathrm{~s}$ following TE (POST-60). The same individual who auscultated the dogs did so from the same position outside of the treadmill for the PRE, WALK, and POST-60 timepoints. Each of the TE sessions was $6 \mathrm{~min}$ in duration with a speed of $0.67 \mathrm{~m} / \mathrm{s}$ (for a total of $239.57 \mathrm{~m}$ ). This speed was selected since it is the speed used in the rehabilitation practice for any patient of this size who has TE as part of their therapeutic regimen. Dogs completed this protocol three times in 1 day.

All three HR monitoring methods were used for REST, PRE, and POST-60. Only the HOLTER and HR MONITOR collections were accomplishable while walking (due to the noise from the fur movement under the stethoscope bell obscuring the heart sounds) and were recorded at POST-15. Real-time video recordings were made of the screens of both monitors. HR was auscultated over the course of a minute to count the heartbeats, as the HR obtained over $15 \mathrm{~s}$ has been reported to have a 2$5 \mathrm{bpm}$ difference from HR counted over $60 \mathrm{~s}(48,49)$. HOLTER transmitted the HR data via Bluetooth to a nearby laptop. The HR MONITOR transmitted the data via Bluetooth to a nearby iPhone 7S. HR from the HOLTER was determined by manually

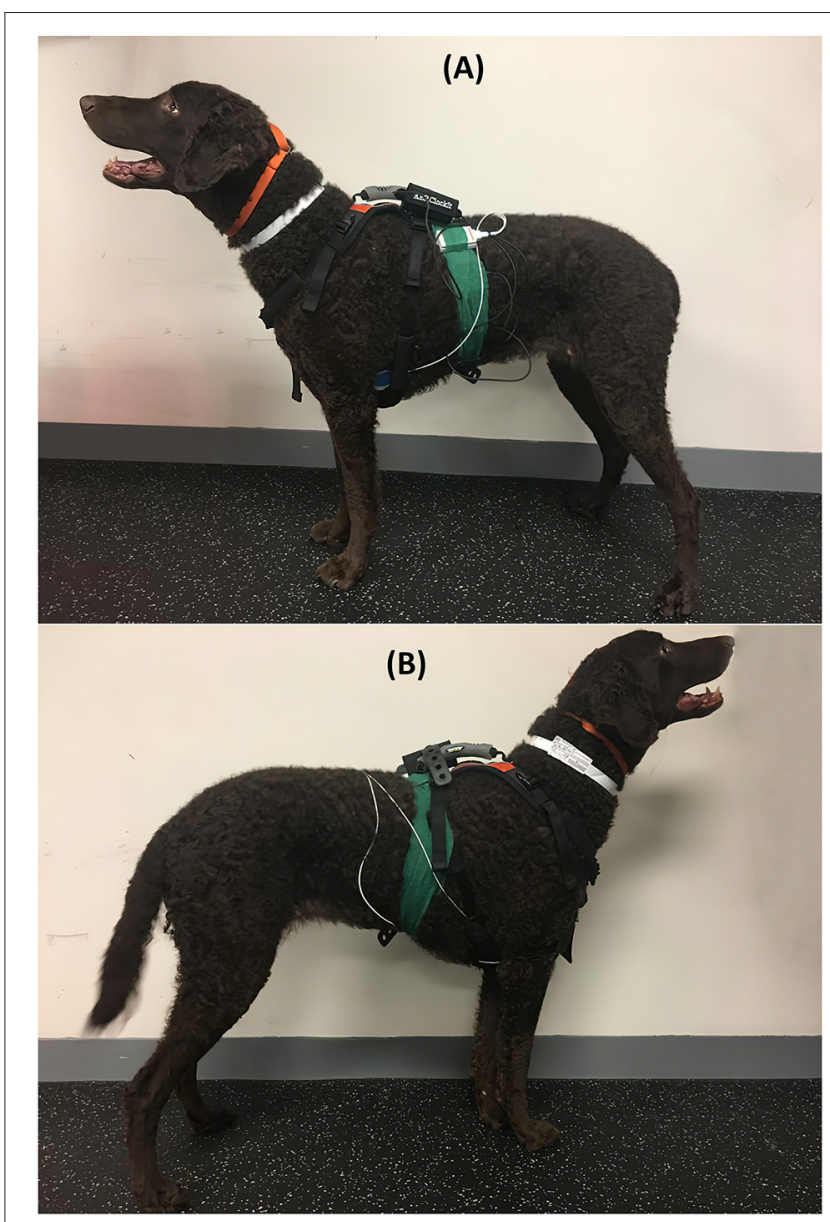

FIGURE 1 | Dog equipped with both the HOLTER and HR MONITOR monitors from the left side (A), where both the HOLTER and HR MONITOR monitors have pads, and from the right side (B), where only HOLTER has pads.

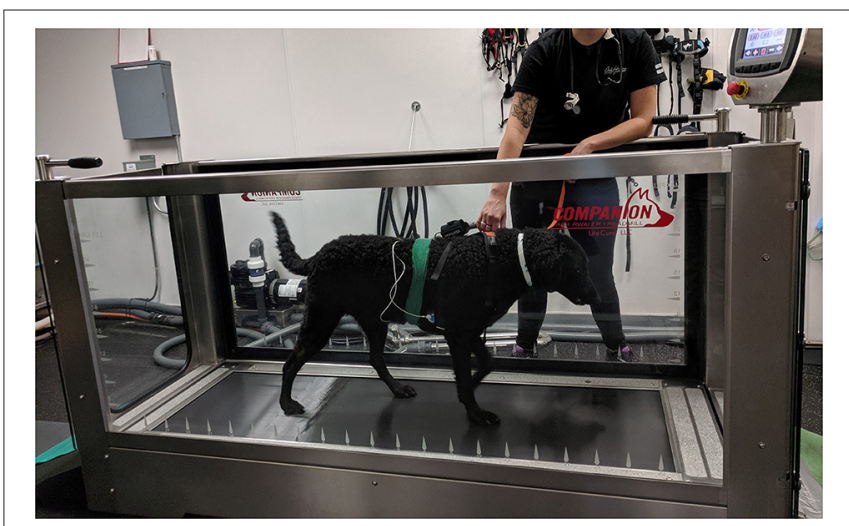

FIGURE 2 | Dog equipped with both monitors while on the treadmill.

counting the QRS waves recorded in CardioExplorer (Dextronix, Sacramento, CA, USA). For the HR MONITOR, the numbers present on the video recording of the iPhone screen at 0,15 , 
TABLE 1 | Mean ( \pm SD) HR of all monitoring options at different timepoints $(N=$ 8 dogs).

\begin{tabular}{lccccc}
\hline Monitoring option & REST & PRE & WALK & POST-15 & POST-60 \\
\hline Auscultation & $102(9) \mathrm{a}$ & $98(8) \mathrm{a}$ & $\mathrm{N} / \mathrm{A}$ & $\mathrm{N} / \mathrm{A}$ & $98(8) \mathrm{a}$ \\
HOLTER & $103(10) \mathrm{a}$ & $96(7) \mathrm{a}$ & $117(1.5) \mathrm{b}$ & $103(9) \mathrm{a}$ & $99(8) \mathrm{a}$ \\
HR MONITOR & $103(6) \mathrm{a}$ & $102(15) \mathrm{a}$ & $121(5) \mathrm{b}$ & $107(14) \mathrm{a}$ & $108(12) \mathrm{a}$
\end{tabular}

HR during WALK was not possible to obtain with auscultation. Different letters indicate differences within a row between timepoints. N/A indicates not able to be obtained.

30,45 , and $60 \mathrm{~s}$ during the minute of interest were averaged to determine the HR.

\section{Statistical Analyses}

Statistical analyses included the Shapiro-Wilk test for normality assessment, Spearman or Pearson correlation coefficients [with a correlation of $>0.7$ being desirable (22)], Bland-Altman plots with 95\% limits of agreement (the HOLTER monitor, which is based on ECG recordings, was considered as the gold standard) to investigate bias (means/difference), and repeated measures ANOVAs with Sidak's post-hoc test (significant at $p<0.05$ ). An a priori sample size of eight dogs was determined with an alpha of $0.05,80 \%$ power, a mean difference of 20 beats, and a SD of 10 (http://openepi.com/Menu/OE_Menu.htm).

\section{RESULTS}

\section{The Effect of TE and HR Monitoring Feasibility}

The mean $( \pm S D)$ HRs for monitoring options at all timepoints are found in Table 1. Auscultation during exercise (WALK) was not possible due to the sound of hair movement muffling the heart sounds. TE significantly increased HR (mean increase of $20 \mathrm{bpm} \pm 4.3, p<0.05)$ during WALK as compared to PRE, REST, POST-15, and POST-60 in both the HOLTER and the HR MONITOR (Table 1). Elevations in HR during WALK were not reflected in the POST-15 or POST-60 timepoints. Peak HR between the three WALK sessions was not significantly different $(P=0.29)$.

The HOLTER could not capture instantaneous HR, which was known before the start of the study, and this did not affect our ability to upload the data and assess HR by counting the QRS waves in the proprietary software. The HR MONITOR would not always upload the HR during the sessions after their completion to the cloud, which was known before the start of the study and which was not an issue due to the screen video recordings.

\section{Comparison of HR Agreement for All HR Monitoring Options}

Significant strong correlations existed between HOLTER and HR MONITOR at REST and WALK (both $r h o=0.9, p<0.01$ ) and between the HOLTER and auscultation at REST, PRE, and POST60 (all rho $=0.9, p<0.01$ ). There was a significant moderate correlation between HOLTER and HR MONITOR at PRE (rho $=0.75, p<0.001$ ) and POST-15 (rho $=0.72, p<0.001)$. Auscultation and HR MONITOR moderately correlated at PRE
Difference vs. average: Bland-Altman REST HOLTER vs HR MONITOR

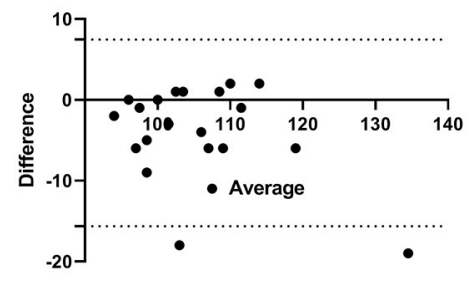

Difference vs. average: Bland-Altman WALK HOLTER vs. HR MONITOR

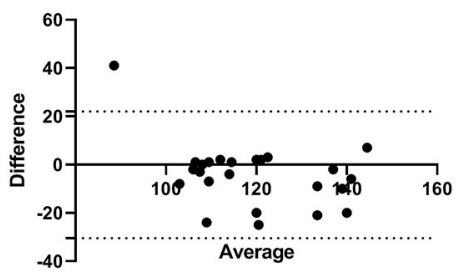

FIGURE 3 | The Bland-Altman plots show the agreement between the HOLTER and HR MONITOR monitors during HR measurement at REST (top) and WALK (bottom). The $X$ axis is the average of the HOLTER and HR MONITOR, and the $Y$ axis shows the mean difference between the methods. The dotted lines represent the upper and lower 95\% limits of agreement.

$(r h o=0.5, P=0.01)$ and POST-60 $(r h o=0.55, p<0.001)$ but had a significant strong correlation at REST (rho $=0.9, P$ $=0.002$ ).

Bland-Altman analyses of HOLTER vs. HR MONITOR showed all but two data points at REST, and one data point at WALK was within the 95\% limits of agreement (Figure 3), although there was a bias for the HR MONITOR to give higher HR readings than HOLTER during both REST (bias $=-4.1$ ) and WALK (bias $=-4.2$ ).

\section{Examination for Correlations Between Peak HR and Age and Peak HR and Weight}

Peak HR did not statistically correlate with the age $\left(r^{2}=0.001, P\right.$ $=0.94)$ or body weight of the $\operatorname{dog}\left(r^{2}=0.14, P=0.36\right)$.

\section{DISCUSSION}

The hypothesis that the HR could be readily measured before, during, and after a TE session using a HR monitor was accepted, noting that different monitors may be better for different applications. The hypotheses that HR is elevated during walk exercise as compared to rest in healthy dogs and that postexercise HR measurements do not accurately demonstrate the HR achieved during exercise were accepted. Relying on postexercise auscultation alone could underestimate workload, fear, and pain, leading to increased patient risk of injury.

Boddy et al. (20) and Swimmer and Rozanski (21) found no difference between pre-exercise $H R$ values and post-exercise HR values in healthy dogs. Similarly, in the present study, no monitoring technique found a difference between the preexercise and post-exercise measurements. In Boddy et al. (20) any HR elevations during the exercise may have been missed as 
HR was assessed only once the dog stopped and not while the dog was still exercising. Swimmer and Rozanski (21) found that the dogs affected by pulmonary disease had an elevated postexercise $\mathrm{HR}$ value despite walking less distance than normal dogs, but the true extent of HR elevation cannot be known as HR during exercise was not assessed. In a study that measured HR during a trot, Essner et al. (26) found an increase in HR during activity from baseline $(124 \pm 15.6 \mathrm{bpm}$ from a baseline of 86 bpm). The current study also saw an increase in HR with walk exercise, though as expected the change in HR was less robust [20 bpm difference vs. $\sim 38 \mathrm{bpm}$ in the study of Essner et al. (26)] due to the slower gait. In the current study, the HR monitors did allow for HR measurement during TE (WALK), which was significantly higher than the rest, pre- and both post-exercise measurements. This would support a greater need to identify intra-exercise HR values and for the establishment of normal parameters in healthy animals.

The unexpectedly rapid return to pre-exercise HR levels could be due to the high vagal tone of healthy animals undergoing light exercise. A fast HR recovery at $10 \mathrm{~s}$ after cessation of exercise has been linked to lower mortality and is attributed to the reactivation of the parasympathetic nervous system while the sympathetic nervous system withdraws (45). In humans and dogs with cardiac disease, high-intensity interval training sped up HR recovery, and a faster HR recovery led to less risk for ventricular fibrillation (41, 42). Dogs with induced cardiac disease that underwent prolonged submaximal exercise regained higher vagal tone and had lower HRs post-exercise than sedentary dogs (43). It is possible that the healthy dogs in this study had higher levels of vagal tone due to their regular 4 days a week of brisk walk activity such that their return to baseline HR post-exercise was rapid. While athleticism can ensure a fast HR recovery, the intensity of exercise can override vagal tone. For instance, in racing greyhounds undergoing high-intensity exercise $(\mathrm{a} 100 \mathrm{~m}$ sprint), the elevation of HR was seen in the immediate postexercise auscultation (30). Most rehabilitation patients are not as fit as either the normal dogs in the current study or the racing greyhounds in the previously mentioned study. HR elevations during exercise and afterward would be predicted in all dogs, but the amount likely depends on the dog's health status and the type of exercise being performed.

Peak HR did not correlate with the age or the body weight of the dogs in this study. Previous work by Hezzell et al. (50) found that body weight, but not age, had a negative association with HR. A $50 \mathrm{~kg}$ decrease in weight was only associated with a $10.5 \mathrm{bpm}$ increase in HR so it was not unexpected that in the current study, where the dogs were close in body weight, no association was seen. While age was significant in their initial univariate analysis, having a positive association with $\mathrm{HR}$, it was not significant in their final model, nor was it significant in this study (50).

In this study, the investigators did identify some peculiarities in the management of the HR monitoring systems. During the study, the HR MONITOR software would not consistently upload via the cloud for storage and would not permanently store it. This was deemed a network and software issue that could be overcome. The HOLTER did not display instantaneous singlenumber HR values on the screen in real time. It was believed that this was a software setting that could be updated. Both of these issues were known prior to this study, and our methodology allowed for appropriate data collection and analysis. The data that were collected for both systems did correlate with the auscultated values collected. The harness and tape assembly used to secure the system of the dog was applied quickly with minimal effort, allowing the dogs to move freely. Thus, either of these monitors could be used to evaluate the HR during commonly performed therapeutic exercises, such as cavalettis and balance work or during underwater TE (the latter has been performed in our clinic with water at shoulder height with the HOLTER). Although it is possible submersion in the treadmill water could alter measured HR, the HOLTER monitor has already been used to investigate HR in diving seals $(46,47)$, suggesting that submersion in treadmill water would not be an issue.

Heart rate monitoring agreement was good to excellent between the methods and similar to previous reports $(20,21)$, indicating that either the HOLTER or HR MONITOR could be used in practice to provide insight into HR changes with walk exercise. The slight overestimation of HR in the HR MONITOR suggests that the HOLTER would still be preferred if available. Auscultation has been replaced by Holter monitoring of HR if possible in human research studies as the former has been found to be inaccurate (51). The limitations of this study included that only healthy, non-obese, large breed dogs were used and that the monitors were only assessed for HRs of up to $150 \mathrm{bpm}$ at one speed. Normal HR variability and recovery with exercise should be investigated in medium and small breed dogs as has been done with swimming exercise (30).

Real-time HR monitoring appears to be feasible in rehabilitation practice, and the results of this study can offer some guidelines for use. While exercising at $0.67 \mathrm{~m} / \mathrm{s}$, a speed appropriate for walk rehabilitative TE, it was necessary to evaluate the HR during motion, as HR measurement postactivity did not reflect the elevated HR in healthy dogs while walking on a treadmill. Future studies at other paces, during other exercises, and in patients undergoing rehabilitation would be encouraged to record HR during the actual activity as well. The HOLTER, should allow updates for the projection of real-time instantaneous HR numbers on the monitor, which would be good for practice or research, but in its current form, it is more appropriate for research. The HR MONITOR would be appropriate for practice as it offers real-time instantaneous HR assessment, but is less than ideal for research as its software limitations require additional steps to analyze. Developing normal ranges for HR parameters during rehabilitation exercises can guide post-operative TE regimens to minimize patient risk of injury and to maximize the efficiency of recovery and training effectiveness. Exceeding a $20 \%$ increase in HR during walk TE in a large-sized dog could indicate that they are exceeding their conditioning (which could lead to fatigue and injury) or that they are under increased pain or physiologic stress which could be mitigated by adjusting the intensity or duration of the TE. Further study of HR during TE in populations of animals with various cardiovascular, musculoskeletal, and neurologic conditions is warranted. 


\section{DATA AVAILABILITY STATEMENT}

The raw data supporting the conclusions of this article will be made available by the authors, without undue reservation.

\section{ETHICS STATEMENT}

The animal study was reviewed and approved by Michigan State University's Institutional Animal Care and Use Committee. Written informed consent was obtained from the owners for the participation of their animals in this study.

\section{REFERENCES}

1. Astrand PO, Saltin B. Oxygen uptake during the first minutes of heavy muscular exercise. J Appl Physiol. (1961) 16:9716. doi: 10.1152/jappl.1961.16.6.971

2. Åstrand P-O, Saltin B. Maximal oxygen uptake and HR in various types of muscular activity. J Appl Physiol. (1961) 16:977-81. doi: 10.1152/jappl.1961.16.6.977

3. Lester M, Sheffield L, Trammell P, Reeves T. The effect of age and athletic training on the maximal HR during muscular exercise. Am Heart J. (1968) 76:370-6. doi: 10.1016/0002-8703(68)90233-0

4. Achten J, Jeukendrup AE. HR monitoring. Sports Med. (2003) 33:51738. doi: 10.2165/00007256-200333070-00004

5. Bourdon PC, Cardinale M, Murray A, Gastin P, Kellmann M, Varley MC, et al. Monitoring athlete training loads: consensus statement. Int J Sports Physiol Perform. (2017) 12(Suppl. 2):S2161-S270. doi: 10.1123/IJSPP.2017-0208

6. Chai G, Wang Y, Wu J, Yang H, Tang Z, Zhang L. Study on the recognition of exercise intensity and fatigue on runners based on subjective and objective information. Healthcare. (2019)7:150. doi: 10.3390/healthcare7040150

7. Khan Y, Ostfeld AE, Lochner CM, Pierre A, Arias AC. Monitoring of vital signs with flexible and wearable medical devices. Adv Mater. (2016) 28:437395. doi: 10.1002/adma.201504366

8. Laukkanen RM, Virtanen PK. HR monitors: state of the art. J Sports Sci. (1998) 16(suppl. 1):3-7. doi: 10.1080/026404198366920

9. Shruthi P, Resmi R, editors. HR monitoring using pulse oximetry and development of fitness application. In: 2019 2nd International Conference on Intelligent Computing, Instrumentation and Control Technologies (ICICICT): Kannur: IEEE (2019). doi: 10.1109/ICICICT46008.2019.8993398

10. Association APT. Guide to Physical Therapist Practice 3.0. Alexandria, VA: American Physical Therapy Association (2014).

11. Frese EM, Richter RR, Burlis TV. Self-reported measurement of HR and blood pressure in patients by physical therapy clinical instructors. Phys Ther. (2002) 82:1192-200. doi: 10.1093/ptj/82.12.1192

12. Millar AL, Village D, King T, McKenzie G, Lee J, Lopez C. HR and blood pressure assessment by physical therapists in the outpatient setting -an observational study. Cardiopulm Phys Ther J. (2016) 27:905. doi: $10.1097 /$ CPT.0000000000000033

13. McCauley L, Van Dyke JB. Therapeutic exercise. Canine Sports Medicine and Rehabilitation. Hoboken, NJ: John Wiley and Sons, Inc. (2018). p. 177207. doi: $10.1002 / 9781119380627 . c h 8$

14. Saunders DG. Therapeutic exercise. Clin Tech Small Anim Pract. (2007) 22:155-9. doi: 10.1053/j.ctsap.2007.09.003

15. Zink MC, Van Dyke JB, Wiley J. Canine Sports Medicine and Rehabilitation. Ames, IA: Wiley Online Library (2013). doi: 10.1002/9781118783443

16. Bloomberg MS, Dee JF, Taylor RA. Canine Sports Medicine and Surgery. St. Louis, MO: Saunders (1998).

17. Blythe LL, Gannon J, Craig A, Fegan D. Care of the Racing \& Retired Greyhound. Dostupné z. (2007). Available online at: http://www.dogracing.cz/ upload/greyhound_krev_pdf (accessed November 11, 2020).

\section{AUTHOR CONTRIBUTIONS}

SS contributed to research idea, study design, data collection and analysis, and manuscript preparation. SR contributed to study design, data collection and analysis, and manuscript preparation. RG and JM contributed to study design, data analysis, and manuscript preparation. All authors contributed to the article and approved the submitted version.

\section{FUNDING}

This work was supported by Michigan State University College of Veterinary Medicine Institutional Funds (Shull).

18. Dirsko von Pfeil JL, Thompson S, Hinchcliff K. Musher and Veterinary Handbook. 3rd ed. Washington, DC: International Sled Dog Veterinary Medical Association (2015).

19. Swanson K, Harper TAM, McMichael M, Fries RC, Lascola K, Chandler C, et al. Development of a perceived exertion scale for dogs using selected physiologic parameters. J Small Anim Pract. (2019) 60:24753. doi: $10.1111 /$ jsap. 12978

20. Boddy KN, Roche BM, Schwartz DS, Nakayama T, Hamlin RL. Evaluation of the six-minute walk test in dogs. Am J Vet Res. (2004) 65:3113. doi: 10.2460/ajvr.2004.65.311

21. Swimmer RA, Rozanski EA. Evaluation of the 6 minute walk test in pet dogs. J Vet Intern Med. (2011) 25:405-6. doi: 10.1111/j.1939-1676.2011.0689.x

22. Carter R, Lubinsky J. Rehabilitation Research: Principles and Applications. St. Louis, MO: Elsevier Health Sciences (2015).

23. Crosara S, Borgarelli M, Perego M, Häggström J, La Rosa G, Tarducci A, et al. Holter monitoring in 36 dogs with myxomatous mitral valve disease. Aus Vet J. (2010) 88:386-92. doi: 10.1111/j.1751-0813.2010.00628.x

24. Del Rio CL, Clymer BD, Billman GE. Myocardial electrotonic response to submaximal exercise in dogs with healed myocardial infarctions: evidence for $\beta$-adrenoceptor mediated enhanced coupling during exercise testing. Front Physiol. (2015) 6:25. doi: 10.3389/fphys.2015.00025

25. Rasmussen CE, Falk T, Domanjko Petrič A, Schaldemose M, Zois NE, Moesgaard S, et al. Holter monitoring of small breed dogs with advanced myxomatous mitral valve disease with and without a history of syncope. J Vet Int Med. (2014) 28:363-70. doi: 10.1111/jvim. 12290

26. Essner A, Sjöström R, Ahlgren E, Lindmark B. Validity and reliability of Polar $\AA$ RS800CX HR monitor, measuring HR in dogs during standing position and at trot on a treadmill. Physiol Behav. (2013) 114:15. doi: 10.1016/j.physbeh.2013.03.002

27. Jonckheer-Sheehy VS, Vinke CM, Ortolani A. Validation of a Polar $($ human $\mathrm{HR}$ monitor for measuring $\mathrm{HR}$ and $\mathrm{HR}$ variability in adult dogs under stationary conditions. J Vet Behav. (2012) 7:205-12. doi: 10.1016/j.jveb.2011.10.006

28. Gillette RL, Angle TC, Sanders JS, DeGraves FJ. An evaluation of the physiological affects of anticipation, activity arousal and recovery in sprinting Greyhounds. Appt Anim Behav Sci. (2011) 130:101-6. doi: 10.1016/j.applanim.2010. 12.010

29. Hydbring-Sandberg E, von Walter LW, Hoglund K, Svartberg K, Swenson L, Forkman B. Physiological reactions to fear provocation in dogs. J Endo. (2004) 180:439-48. doi: 10.1677/joe.0.1800439

30. Pellegrino FJ, Risso A, Vaquero PG, Corrada YA. Physiological parameter values in greyhounds before and after high-intensity exercise. Open Vet J. (2018) 8:64-7. doi: 10.4314/ovj.v8i1.11

31. Piccione G, Casella S, Panzera M, Giannetto C, Fazio F. Effect of moderate treadmill exercise on some physiological parameters in untrained beagle dogs. Exp Anim. (2012) 61:511-5. doi: 10.1538/expanim.61.511

32. Rizzo M, Arfuso F, Alberghina D, Giudice E, Gianesella M, Piccione G. Monitoring changes in body surface temperature associated with treadmill 
exercise in dogs by used of infrared methodology. J Therm Bio. (2017) 69:64-8. doi: 10.1016/j.jtherbio.2017.06.007

33. Benson GJ, Grubb TL, Neff-Davis C, Olson WA, Thurmon JC, Lindner DL, et al. Perioperative stress response in the dog: effect of pre-emptive administration of medetomidine. Vet Surg. (2000) 29:85-91. doi: 10.1111/j.1532-950X.2000.00085.x

34. Task Force of the European Society of Cardiology the North American Society of Pacing Electrophysiology. HR Variability. Circulation. (1996) 93:104365. doi: 10.1161/01.CIR.93.5.1043

35. Gouin J-P, Kiecolt-Glaser JK. The impact of psychological stress on wound healing: methods and mechanisms. Immuno Allergy Clin. (2011) 31:8193. doi: 10.1016/j.iac.2010.09.010

36. Hallman DM, Lyskov E. Autonomic regulation, physical activity and perceived stress in subjects with musculoskeletal pain: 24hour ambulatory monitoring. Int J Psychophys. (2012) 86:27682. doi: 10.1016/j.ijpsycho.2012.09.017

37. Soon K, Acton C. Pain-induced stress: a barrier to wound healing. Wounds UK. (2006) 2:92.

38. Walburn J, Vedhara K, Hankins M, Rixon L, Weinman J. Psychological stress and wound healing in humans: a systematic review and metaanalysis. J Psychosom Res. (2009) 67:253-71 doi: 10.1016/j.jpsychores.2009. 04.002

39. Shetler K, Marcus R, Froelicher VF, Vora S, Kalisetti D, Prakash M et al. Heart rate recovery: validation and methodologic issues. J Am Coll Cardiol. (2001) 38:1980-7. doi: 10.1016/S0735-1097(01)01652-7

40. Peçanha T, Silva-Júnior ND, de Moraes Forjaz CL. Heart rate recovery: autonomic determinants, methods of assessment and association with mortality and cardiovascular diseases. Clin Physiol Funct Imaging. (2014) 34:327-39. doi: 10.1111/cpf.12102

41. Villelabeitia-Jaureguizar K, Vicente-Campos D, Senen AB, Jiménez $\mathrm{AH}$, Garrido-Lestache MEB, Chicharro JL. Effects of high-intensity interval versus continuous exercise training on post-exercise heart rate recovery in coronary heart-disease patients. Int J Cardiol. (2017) 244:17-23. doi: 10.1016/j.ijcard.2017.06.067

42. Smith LL, Kukielka M, Billman, GE. Heart rate recovery after exercise: a predictor of ventricular fibrillation susceptibility after myocardial infarction. Am $J$ Physiol Heart Circ Physiol. (2005) 288:H1763-9. doi: 10.1152/ajpheart.00785. 2004

43. Billman GE and M Kukielka. Effect of endurance exercise training on heart rate onset and heart rate recovery responses to submaximal exercise in animals susceptible to ventricular fibrillation. J Appl Physiol. (2007) 102:23140. doi: 10.1152/japplphysiol.00793.2006

44. Kukielka M, Seals D, Billman GE. Cardiac vagal modulation of heart rate during prolonged submaximal exercise in animals with healed myocardial infarctions: effects of training. Am J Physiol Heart Circ Physiol. (2006) 290:H1680-5. doi: 10.1152/ajpheart.01034.2005

45. van de Vegte YJ, van der Harst P, Verweij N. Heart rate recovery 10 seconds after cessation of exercise predicts death. J Am Heart Assoc. (2018) 7:e008341. doi: 10.1161/JAHA.117.008341

46. Greaves DK, Schreer JF, Hammill MO, Burns JM. Diving heart rate development in postnatal harbour seals, Phoca vitulina. Physiol Biochem Zoo. (2005) 78:9-17. doi: 10.1086/425201

47. Fonfara S, Casamian-Sorrosal D, Sundermeyer J, Rosenberger T. Variations in heart rate and rhythm of harbor seal pups during rehabilitation. Mar Mamm Sci. (2015) 31:998-1013. doi: 10.1111/mms.12201

48. Hollerbach AD, Sneed NV. Accuracy of radial pulse assessment by length of counting interval. Heart Lung. (1990) 19:258-64.

49. Kobayashi H. Effect of measurement duration on accuracy of pulse-counting. Ergonomics. (2013) 56:1940-4. doi: 10.1080/00140139.2013.840743

50. Hezzell MJ, Humm K, Dennis SG, Agee L, Boswood A. Relationships between heart rate and age, bodyweight and breed in 10,849 dogs. J Small Anim Pract. (2013) 54:318-24. doi: 10.1111/jsap.12079

51. Murphy MC, De Angelis L, McCarthy LK, O’Donnell CPF. Comparison of infant HR assessment by auscultation, ECG and oximetry in the delivery room. Arch Dis Child Fetal Neonatal Ed. (2018) 103:F490F492. doi: 10.1136/archdischild-2017-314367

Conflict of Interest: RG was employed by company Sportsvet Veterinary Consulting Services.

The remaining authors declare that the research was conducted in the absence of any commercial or financial relationships that could be construed as a potential conflict of interest.

Copyright (c) 2021 Shull, Rich, Gillette and Manfredi. This is an open-access article distributed under the terms of the Creative Commons Attribution License (CC BY). The use, distribution or reproduction in other forums is permitted, provided the original author(s) and the copyright owner(s) are credited and that the original publication in this journal is cited, in accordance with accepted academic practice. No use, distribution or reproduction is permitted which does not comply with these terms. 